\title{
On the solvability of nonlinear elliptic equations in Sobolev spaces
}

\author{
by Piotr FijaŁkowski (Łódź)
}

Abstract. We consider the existence of solutions of the system $(*)$

$$
P(D) u^{l}=F\left(x,\left(\partial^{\alpha} u\right)\right), \quad l=1, \ldots, k, x \in \mathbb{R}^{n}
$$

$\left(u=\left(u^{1}, \ldots, u^{k}\right)\right)$ in Sobolev spaces, where $P$ is a positive elliptic polynomial and $F$ is nonlinear.

1. Introduction. We study the existence of solutions of the system (*) in Sobolev spaces. We make such assumptions that the right sides of the considered equations are locally integrable for $u$ belonging to a space of solutions. In this way, we can understand these equations in the sense of distributions.

The other assumptions concerning the right sides of equations $(*)$ give a priori bounds of solutions. We consider, for example, assumptions of the Bernstein type. Assumptions of this kind can be found in the papers [1], [4] concerning equations on a bounded interval, in [8] concerning equations on the half-line and in [3] concerning equations on the line.

We shall denote by $\langle\cdot, \cdot\rangle$ the scalar product and by $|\cdot|$ the euclidean norm in $\mathbb{R}^{l}$ for any positive integer $l$.

The Fourier transform of $f \in L^{1}\left(\mathbb{R}^{n}\right)$ is defined by

$$
(\mathcal{F} f)(\xi):=\int e^{-i\langle x, \xi\rangle} f(x) d x,
$$

where $\int=\int_{\mathbb{R}^{n}}$. We define the Fourier transformation in the space of tempered distributions in the standard way.

By $\mathcal{H}^{s}=\mathcal{H}^{s}\left(\mathbb{R}^{n}\right)$, for real $s \geq 0$, we denote the real Sobolev space of real tempered distributions $u$ such that

$$
\|u\|_{s}^{2}:=(2 \pi)^{-n} \int|(\mathcal{F} u)(\xi)|^{2}\left(1+|\xi|^{2}\right)^{s} d \xi<\infty .
$$


(We have $\mathcal{H}^{0}=L^{2}=L^{2}\left(\mathbb{R}^{n}\right)$.) We denote the local Sobolev space by $\mathcal{H}_{\text {loc }}^{s}=\mathcal{H}_{\text {loc }}^{s}\left(\mathbb{R}^{n}\right)$ and treat it as a Fréchet space in the standard way (see for example [5]).

We denote the space of $\mathcal{C}^{\infty}$-functions on $\mathbb{R}^{n}$ with compact support by $\mathcal{C}_{0}^{\infty}$ and the space of Schwartz distributions on $\mathbb{R}^{n}$ by $\mathcal{D}^{\prime}$.

By $\alpha=\left(\alpha_{1}, \ldots, \alpha_{n}\right)$ we denote a multi-index, $|\alpha|:=\sum_{i=1}^{n} \alpha_{i}$. We set $\partial_{j}:=\partial / \partial x_{j}$ and $D_{j}:=-i \partial_{j}$.

2. Existence theorem for a single equation. We prove the following

THEOREM 1. Let $P$ be a polynomial of $n$ variables and degree $T$ such that the polynomial $P(-i \partial)$ of the variable $\partial$ has real coefficients. Assume that

$$
1+|\xi|^{T} \leq C|P(\xi)|, \quad \xi \in \mathbb{R}^{n}
$$

for some constant $C$.

Let $t \in[0, T[$ and

$$
m:=\sum_{0 \leq l \leq t} n^{l}, \quad m^{\prime}:=\sum_{0 \leq l<t-n / 2} n^{l},
$$

where $l$ is an integer variable. (We set $\sum_{l \in \emptyset} n^{l}:=0$.)

Suppose that $F: \mathbb{R}^{n} \times \mathbb{R}^{m} \rightarrow \mathbb{R}$ satisfies the Carathéodory condition: $F(x, \cdot)$ is continuous for almost all $x \in \mathbb{R}^{n}$ and $F\left(\cdot,\left(v_{\alpha}\right)_{|\alpha| \leq t}\right)$ is measurable for all $\left(v_{\alpha}\right)_{|\alpha| \leq t} \in \mathbb{R}^{m}$.

For any compact set $K \subset \mathbb{R}^{n} \times \mathbb{R}^{m^{\prime}}$, let there exist a function $h_{K} \in$ $L^{2}\left(\mathbb{R}^{n}\right)$ and a constant $C_{K}$ such that

$$
\left|F\left(x,\left(v_{\alpha}\right)_{|\alpha| \leq t}\right)\right| \leq h_{K}(x)+C_{K}\left|\left(v_{\alpha}\right)_{t-n / 2 \leq|\alpha| \leq t}\right|
$$

for all $\left(x,\left(v_{\alpha}\right)_{|\alpha|<t-n / 2}\right) \in K$ almost everywhere with respect to $x$ (a.e. $\left.x\right)$. (We omit the last term in (2) if $m=m^{\prime}$.)

Suppose that there exist a sequence of open bounded sets $U_{1} \subset U_{2} \subset \ldots$, $\bigcup U_{j}=\mathbb{R}^{n}$, and a constant $M$ such that any equation

$$
\begin{gathered}
P(D) u=\lambda F_{j}\left(x,\left(\partial^{\alpha} u\right)_{|\alpha| \leq t}\right), \quad j=1,2, \ldots, \lambda \in[0,1], \\
F_{j}\left(x,\left(v_{\alpha}\right)_{|\alpha| \leq t}\right):= \begin{cases}F\left(x,\left(v_{\alpha}\right)_{|\alpha| \leq t}\right) & \text { for } x \in U_{j}, \\
0 & \text { for } u \notin U_{j},\end{cases}
\end{gathered}
$$

has no solution in the set $\left\{u \in \mathcal{H}^{t}:\|u\|_{t}>M\right\}$.

Under these assumptions, the equation

$$
P(D) u=F\left(x,\left(\partial^{\alpha} u\right)_{|\alpha| \leq t}\right)
$$

has a solution $u$ in $\mathcal{H}^{t}$ for which $\|u\|_{t} \leq M$. 
The proof of Theorem 1 is based on several lemmas.

LEMmA 1. If $u \in \mathcal{H}^{s}$, then any $\partial^{\alpha} u$, for $|\alpha|<s-n / 2$, is a continuous bounded function and there exists a constant $C$ such that

$$
\sup _{x \in \mathbb{R}^{n}} \sup _{|\alpha|<s-n / 2}\left|\partial^{\alpha} u(x)\right| \leq C\|u\|_{s} .
$$

Pr o of. See [5], Corollary 7.9.4. One can obtain inequality (5) by standard calculus.

LEMma 2. The Nemytskiu operator $u \mapsto F_{j}\left(\cdot,\left(\partial^{\alpha} u(\cdot)\right)_{|\alpha| \leq t}\right)$ transforms $\mathcal{H}_{\text {loc }}^{t}$ into $L^{2}$ continuously.

Proof. If $u \in \mathcal{H}_{\text {loc }}^{t}$, then $F_{j}\left(\cdot,\left(\partial^{\alpha} u(\cdot)\right)_{|\alpha| \leq t}\right)$ is measurable by the Carathéodory condition (see [2], appendix, or $[6], \S 17)$. The set

$$
K:=\bar{U}_{j} \times \underset{|\alpha|<t-n / 2}{\chi} \partial^{\alpha} u\left(\bar{U}_{j}\right)
$$

is compact because of the continuity of $\partial^{\alpha} u$ for $|\alpha|<s-n / 2$ due to Lemma 1 . We have $F_{j}\left(\cdot,\left(\partial^{\alpha} u(\cdot)\right)_{|\alpha| \leq t}\right) \in L^{2}$ by (2) for $K$ defined above. The required continuity is now proved as in [2], appendix, where the case $s=0$ is considered.

Observe that, in $\mathcal{H}^{t}$, equation (3) is equivalent to

$$
u=\lambda A_{j} u,
$$

where

$$
A_{j} u:=\mathcal{F}^{-1}\left(\frac{1}{P} \mathcal{F} F_{j}\left(\cdot,\left(\partial^{\alpha} u(\cdot)\right)_{|\alpha| \leq t}\right)\right) .
$$

DeFinition 1. A continuous operator between locally convex spaces is called completely continuous if it sends bounded sets into precompact ones.

The following lemma is very important for the proof of Theorem 1 .

Lemma 3. The embedding $\mathcal{H}_{\mathrm{loc}}^{s} \rightarrow \mathcal{H}_{\mathrm{loc}}^{s^{\prime}}$ for $s>s^{\prime} \geq 0$ is completely continuous.

The proof is in [5], Theorem 10.1.27.

LEMMA 4. The operator $A_{j}$ defined by $(7)$ is completely continuous from $\mathcal{H}^{T}$ into $\mathcal{H}^{T}$.

P r o of. Observe that, for $u \in \mathcal{H}_{\text {loc }}^{t}$,

$$
\left(1+|\xi|^{T}\right) \mathcal{F}\left(A_{j} u\right)(\xi)=b(\xi) \mathcal{F} F_{j}\left(\cdot,\left(\partial^{\alpha} u(\cdot)\right)_{|\alpha| \leq t}\right)(\xi)
$$

where $b(\xi):=(P(\xi))^{-1}\left(1+|\xi|^{T}\right)$ is bounded by $(1)$. 
Consequently, $A_{j} u \in \mathcal{H}^{T}+i \mathcal{H}^{T}$. We have $A_{j} u \in \mathcal{H}^{T}$ because the polynomial $P(-i \partial)$ of the variable $\partial$ has real coefficients.

It follows easily from Lemma 2 and (8) that $A_{j}$ transforms $\mathcal{H}_{\text {loc }}^{t}$ into $\mathcal{H}^{T}$ continuously. But the embedding $\mathcal{H}^{T} \rightarrow \mathcal{H}_{\text {loc }}^{t}$ is completely continuous by Lemma 3, which proves the lemma.

Lemma 5. There exists a constant $M_{j}$ such that $\left\|u_{j \lambda}\right\|_{T} \leq M_{j}$ for any solution $u_{j \lambda}$ of equation (6) for $\lambda \in[0,1]$.

Proof. From the assumption, we have $\left\|u_{j \lambda}\right\|_{t} \leq M$, hence from (5) and (2)

$$
\left\|F_{j}\left(\cdot,\left(\partial^{\alpha} u_{j \lambda}(\cdot)\right)_{|\alpha| \leq t}\right)\right\|_{0} \leq M_{j}^{\prime}
$$

for some constant $M_{j}^{\prime}$. Using (8), we obtain the result.

Lemma 6. Equation (6) has a solution in $\mathcal{H}^{T}$ for $\lambda=1$ and any $j$.

Proof. Write (6) in the form

$$
\left(I-\lambda A_{j}\right) u=0
$$

where $I$ stands for the identity mapping. We treat $I-\lambda A_{j}$ as a mapping from the ball $B\left(0, M_{j}+1\right) \subset \mathcal{H}^{T}$ into $\mathcal{H}^{T}$ and use the Leray-Schauder degree theory (see for instance [7]), since $A_{j}$ is completely continuous by Lemma 4 . From Lemma 5 , we know that $\left(I-\lambda A_{j}\right) u \neq 0$ for $\|u\|_{T}=M_{j}+1$, so for the Leray-Schauder degree we obtain

$$
\operatorname{deg}\left(I-A_{j}, B\left(0, M_{j}+1\right), 0\right)=\operatorname{deg}\left(I, B\left(0, M_{j}+1\right), 0\right)=1 \neq 0 .
$$

Therefore, equation (6) has a solution in $\mathcal{H}^{T}$ for $\lambda=1$.

Lemma 7. The set $\left\{u_{j}\right\}$ of solutions of equations (3), for $\lambda=1$, in the space $\mathcal{H}^{T}$ is bounded in the space $\mathcal{H}_{\mathrm{loc}}^{s}$, where $s:=\min \{t+1, T\}$.

Pro of. Let $\phi \in \mathcal{C}_{0}^{\infty}$. We have to estimate $\left\|\phi u_{j}\right\|_{s}$ by a constant depending on $\phi$ only. From (1) and the Leibniz-Hörmander formula ([5], (1.1.10)), we obtain

$$
\begin{aligned}
& \left\|\phi u_{j}\right\|_{s}=\left((2 \pi)^{-n} \int\left(1+|\xi|^{2}\right)^{s}\left|\mathcal{F}\left(\phi u_{j}\right)(\xi)\right|^{2} d \xi\right)^{1 / 2} \\
& =\left((2 \pi)^{-n} \int\left(1+|\xi|^{2}\right)^{s-T}\left(1+|\xi|^{2}\right)^{T}\left|\mathcal{F}\left(\phi u_{j}\right)(\xi)\right|^{2} d \xi\right)^{1 / 2} \\
& \leq C_{1}\left(\int\left(1+|\xi|^{2}\right)^{s-T}|P(\xi)|^{2}\left|\mathcal{F}\left(\phi u_{j}\right)(\xi)\right|^{2} d \xi\right)^{1 / 2} \\
& \leq C_{1}\left(\int\left(1+|\xi|^{2}\right)^{s-T}\left|\mathcal{F} P(D)\left(\phi u_{j}\right)(\xi)\right|^{2} d \xi\right)^{1 / 2} \\
& \leq C_{1}\left(\int\left(1+|\xi|^{2}\right)^{s-T}\left|\mathcal{F}\left(\sum_{\alpha \in \mathbb{N}^{n}} \partial^{\alpha} \phi\left(\partial^{\alpha} P\right)(D) u_{j} / \alpha !\right)(\xi)\right|^{2} d \xi\right)^{1 / 2}
\end{aligned}
$$




$$
\begin{aligned}
\leq & C_{1}\left(\int\left(1+|\xi|^{2}\right)^{s-T}\left|\mathcal{F}\left(\phi P(D) u_{j}\right)(\xi)\right|^{2} d \xi\right)^{1 / 2} \\
& +C_{1} \sum_{\alpha \neq(0, \ldots, 0)}\left(\int\left(1+|\xi|^{2}\right)^{s-T}\left|\mathcal{F}\left(\partial^{\alpha} \phi\left(\partial^{\alpha} P\right)(D) u_{j} / \alpha !\right)(\xi)\right|^{2} d \xi\right)^{1 / 2}
\end{aligned}
$$

for some constant $C_{1}$. We will estimate the integrals in the last expression:

$$
\begin{aligned}
\int\left(1+|\xi|^{2}\right)^{s-T}\left|\mathcal{F}\left(\phi P(D) u_{j}\right)(\xi)\right|^{2} d \xi & \leq \int\left|\mathcal{F}\left(\phi P(D) u_{j}\right)(\xi)\right|^{2} d \xi \\
& \leq(2 \pi)^{n} \int\left|\phi(x) P(D) u_{j}(x)\right|^{2} d x \\
& =(2 \pi)^{n} \int\left|\phi(x) F_{j}\left(x,\left(\partial^{\alpha} u_{j}(x)\right)_{|\alpha| \leq t}\right)\right|^{2} d x \leq C_{2}
\end{aligned}
$$

for some constant $C_{2}$ depending on $\phi$. In the last step we have used the estimate $\left\|u_{j}\right\|_{t} \leq M$ and (2) for the set

$$
K:=(\operatorname{supp} \phi) \times \underset{|\alpha|<t-n / 2}{\chi}[-M, M] .
$$

Note that the map $\mathcal{H}^{s-T} \ni w \mapsto \psi w \in \mathcal{H}^{s-T}$ is continuous for fixed $\psi \in \mathcal{C}_{0}^{\infty}$ (see [5], Theorem 10.1.15). This implies that, for $\alpha \neq(0, \ldots, 0)$,

$$
\begin{aligned}
\int\left(1+|\xi|^{2}\right)^{s-T} \mid \mathcal{F} & \left.\left(\partial^{\alpha} \phi\left(\partial^{\alpha} P\right)(D) u_{j} / \alpha !\right)(\xi)\right|^{2} d \xi \\
& \leq C_{\alpha} \int\left(1+|\xi|^{2}\right)^{s-T}\left|\mathcal{F}\left(\partial^{\alpha} P\right)(D) u_{j}(\xi)\right|^{2} d \xi \\
& =C_{\alpha} \int\left(1+|\xi|^{2}\right)^{s-T}\left|\left(\partial^{\alpha} P\right)(\xi) \mathcal{F} u_{j}(\xi)\right|^{2} d \xi \\
& =C_{\alpha}^{\prime} \int\left(1+|\xi|^{2}\right)^{s-T}\left|\mathcal{F} u_{j}(\xi)\right|^{2} d \xi \leq C_{\alpha}^{\prime}\left\|u_{j}\right\|_{t} \leq C_{\alpha}^{\prime} M
\end{aligned}
$$

for some constants $C_{\alpha}, C_{\alpha}^{\prime}$ depending on $\phi$. Hence

$$
\left\|\phi u_{j}\right\|_{s} \leq C
$$

for some $C$ depending on $\phi$.

Let $\left(u_{j}\right)$ be a sequence of $\mathcal{H}^{T}$-solutions of equations (3) for $\lambda=1$. The set $\left\{u_{j}\right\}$ is bounded in $\mathcal{H}_{\mathrm{loc}}^{\min \{t+1, T\}}$ by Lemma 7 . Using Lemma 3 , take a subsequence of $\left(u_{j}\right)$ (denoted once more by $\left(u_{j}\right)$ for simplicity of notation) which is convergent to some $u$ in the topology of $\mathcal{H}_{\text {loc }}^{t}$. We have $u \in \mathcal{H}^{t}$ and $\|u\|_{t} \leq M$, since $\left\|u_{j}\right\|_{t} \leq M$.

We shall demonstrate that $u$ is a solution of equation (4). Notice that

$$
F_{j}\left(\cdot,\left(\partial^{\alpha} u_{j}(\cdot)\right)_{|\alpha| \leq t}\right) \rightarrow F\left(\cdot,\left(\partial^{\alpha} u(\cdot)\right)_{|\alpha| \leq t}\right)
$$

in $\mathcal{D}^{\prime}$. Indeed, let $\phi \in \mathcal{C}_{0}^{\infty}$. For large $j$, we have

$$
\begin{aligned}
\int \phi(x) F_{j}\left(x,\left(\partial^{\alpha} u_{j}(x)\right)_{|\alpha| \leq t}\right) d x & =\iint_{\operatorname{supp} \phi} \phi(x) F\left(x,\left(\partial^{\alpha} u_{j}(x)\right)_{|\alpha| \leq t}\right) d x \\
& \rightarrow \int \phi(x) F\left(x,\left(\partial^{\alpha} u(x)\right)_{|\alpha| \leq t}\right) d x .
\end{aligned}
$$

In the last step, we have used Lemma 2. 
The convergence $u_{j} \rightarrow u$ in $\mathcal{H}_{\text {loc }}^{t}$ implies the convergence $u_{j} \rightarrow u$ in $\mathcal{D}^{\prime}$, so also the convergence $P(D) u_{j} \rightarrow P(D) u$ in $\mathcal{D}^{\prime}$. Hence $u$ is a solution of (4).

EXAmple 1. We now define a class of equations for which Theorem 1 is valid.

Assume that $P$ is a real polynomial of degree $T=2 t$, positive for $\xi \in \mathbb{R}^{n}$ and such that the polynomial $P(-i \partial)$ of the variable $\partial$ has real coefficients and (1) is valid.

Let $F: \mathbb{R}^{n} \times \mathbb{R}^{m} \rightarrow \mathbb{R}$ satisfy the Carathéodory condition as above. Let $F$ satisfy (2).

Assume that there exist constants $0<a<2, L>0$ and nonnegative functions $f \in L^{2 / a}, g \in L^{2 /(2-a)}$ such that

$$
v_{(0, \ldots, 0)} F\left(x,\left(v_{\alpha}\right)_{|\alpha| \leq t}\right) \leq 0 \quad \text { if }\left|v_{(0, \ldots, 0)}\right| \geq g(x) \text { a.e. } x,
$$

(11) $\left|F\left(x,\left(v_{\alpha}\right)_{|\alpha| \leq t}\right)\right| \leq f(x)+L\left|\left(v_{\alpha}\right)_{|\alpha| \leq t}\right|^{a} \quad$ if $\left|v_{(0, \ldots, 0)}\right| \leq g(x)$ a.e. $x$.

We show that our assumptions give an a priori bound for solutions of equations (3) where, for example,

$$
F_{j}\left(x,\left(v_{\alpha}\right)_{|\alpha| \leq t}\right)= \begin{cases}F\left(x,\left(v_{\alpha}\right)_{|\alpha| \leq t}\right) & \text { for }|x|<j \\ 0 & \text { for }|x| \geq j .\end{cases}
$$

Let $u=u_{j \lambda} \in \mathcal{H}^{t}$ be a solution of (3). Compute

$$
\begin{aligned}
\|u\|_{t}^{2}= & \int\left(1+|\xi|^{2}\right)^{t}|\mathcal{F} u(\xi)|^{2} d \xi \leq C \int P(\xi) \mathcal{F} u(\xi) \overline{\mathcal{F} u(\xi)} d \xi \\
= & C \int \mathcal{F} u(\xi) \overline{\mathcal{F}(P(D) u)(\xi)} d \xi=(2 \pi)^{n} C \int u(x) \overline{P(D) u(x)} d x \\
= & (2 \pi)^{n} C \int u(x) P(D) u(x) d x \\
= & (2 \pi)^{n} C \lambda \int u(x) F_{j}\left(x,\left(\partial^{\alpha} u(x)\right)_{|\alpha| \leq t}\right) d x \\
\leq & (2 \pi)^{n} C \int u(x) F_{j}\left(x,\left(\partial^{\alpha} u(x)\right)_{|\alpha| \leq t}\right) d x \\
\leq & (2 \pi)^{n} C \int \quad \int \quad u(x) F_{j}\left(x,\left(\partial^{\alpha} u(x)\right)_{|\alpha| \leq t}\right) d x \\
\leq & (2 \pi)^{n} C \quad\{x:|u(x)| \leq g(x)\} \\
\leq & (2 \pi)^{n} C \int g(x)\left(f(x)+L\left|\left(\partial^{\alpha} u(x)\right)_{|\alpha| \leq t}\right|^{a}\right) d x \\
\leq & (2 \pi)^{n} C\left(\int|g(x)|^{2 /(2-a)} d x\right)^{(2-a) / 2}\left(\int|f(x)|^{2 / a} d x\right)^{a / 2} \\
& +(2 \pi)^{n} C L\left(\int|g(x)|^{2 /(2-a)} d x\right)^{(2-a) / 2}\|u\|_{t}^{a} \leq C_{1}\left(1+\|u\|_{t}^{a}\right)
\end{aligned}
$$

for some constant $C_{1}$. In the last steps we have used assumptions (10), (11) and the Hölder inequality. 
Now, it is easy to see that $\|u\|_{t} \leq M$ for some $M$.

3. Existence theorem for a system of equations. We formulate a similar theorem for a system of equations.

TheOREM 2. Let $P_{r}, r=1, \ldots, k$, be polynomials of $n$ variables and degrees $T_{r}$ such that the polynomials $P_{r}(-i \partial)$ of the variable $\partial$ have real coefficients. Assume that

$$
1+|\xi|^{T_{r}} \leq C_{r} P_{r}(\xi), \quad \xi \in \mathbb{R}^{n},
$$

for some constants $C_{r}, r=1, \ldots, k$. Let $t_{r} \in\left[0, T_{r}[\right.$ and

$$
m:=\sum_{r=1}^{k} \sum_{0 \leq l \leq t_{r}} n^{l}, \quad m^{\prime}=\sum_{r=1}^{k} \sum_{0 \leq l<t_{r}-n / 2} n^{l} .
$$

Suppose that $F: \mathbb{R}^{n} \times \mathbb{R}^{m} \rightarrow \mathbb{R}^{k}$ satisfies the Carathéodory condition and, for any compact set $K \subset \mathbb{R}^{n} \times \mathbb{R}^{m}$, there exist a function $h_{K} \in L^{2}\left(\mathbb{R}^{n}\right)$ and a constant $C_{K}$ that

(14) $\left|F\left(x,\left(v_{\alpha}^{r}\right)_{|\alpha| \leq t_{r}, r=1, \ldots, k}\right)\right| \leq h_{K}(x)+C_{K}\left|\left(v_{\alpha}^{r}\right)_{t_{r}-n / 2 \leq|\alpha| \leq t_{r}, r=1, \ldots, k}\right|$ for $\left(x,\left(v_{\alpha}^{r}\right)|\alpha|<t_{r}-n / 2, r=1, \ldots, k\right) \in K$ a.e. $x$. (We omit the last term in (14) if $m=m^{\prime}$.) Suppose that there exist a sequence of open bounded sets $U_{1} \subset$ $U_{2} \subset \ldots, \cup U_{j}=\mathbb{R}^{n}$, and a constant $M>0$ such that the system of equations

$$
P_{l}(D) u^{l}=\lambda F_{j}^{l}\left(x,\left(\partial^{\alpha} u^{r}\right)_{|\alpha| \leq t_{r}, r=1, \ldots, k}\right), \quad l=1, \ldots, k
$$

$\left.\left(F=F^{1}, \ldots, F^{k}\right)\right)$, has no solution in the set

$$
\left\{u=\left(u^{1}, \ldots, u^{k}\right) \in \underset{r=1}{k} \mathcal{H}^{t_{r}}: \sum_{r=1}^{k}\left\|u^{r}\right\|_{t_{r}}^{2}>M^{2}\right\}
$$

for $j=1,2, \ldots, \lambda=[0,1]$. The functions $F_{j}^{l}$ used above are defined by

$$
F_{j}^{l}\left(x,\left(v_{\alpha}^{r}\right)_{|\alpha| \leq t_{r}, r=1, \ldots, k}\right):= \begin{cases}F^{l}\left(x,\left(v_{\alpha}^{r}\right)_{|\alpha| \leq t_{r}, r=1, \ldots, k}\right) & \text { for } x \in U_{j}, \\ 0 & \text { for } x \notin U_{j} .\end{cases}
$$

Under these assumptions, the system of equations

$$
P_{l}(D) u^{l}=F^{l}\left(x,\left(\partial^{\alpha} u^{r}\right)_{|\alpha| \leq t_{r}, r=1, \ldots, k}\right), \quad l=1, \ldots, k,
$$

has a solution $u$ in $\chi_{r=1}^{k} \mathcal{H}^{t_{r}}$ for which

$$
\sum_{r=1}^{k}\left\|u^{r}\right\|_{t_{r}}^{2} \leq M^{2}
$$

We omit the proof, similar to the proof of Theorem 1. 
EXAMPle 2 (cf. Example 1). We define a class of systems for which Theorem 2 is valid.

Assume that $P_{r}, r=1, \ldots, k$, are real polynomials of degrees $T_{r}=2 t_{r}$, positive for $\xi \in \mathbb{R}^{n}$ and such that the polynomials $P_{r}(-i \partial)$ of the variable $\partial$ have real coefficients and (13) is valid. Let $F: \mathbb{R}^{n} \times \mathbb{R}^{m} \rightarrow \mathbb{R}$ satisfy the Carathéodory condition and (14). Assume that there exist constants $0<a<2, L>0$ and nonnegative functions $f \in L^{2 / a}, g \in L^{2 /(2-a)}$ such that

$$
\left\langle v_{(0, \ldots, 0)}, F\left(x,\left(v_{\alpha}^{r}\right)_{|\alpha| \leq t_{r}, r=1, \ldots, k}\right)\right\rangle \leq 0 \quad \text { if }\left|v_{(0, \ldots, 0)}\right| \geq g(x) \text { a.e. } x
$$

$\left(F=\left(F^{1}, \ldots, F^{k}\right)\right)$, and

$$
\begin{aligned}
\left|F\left(x,\left(v_{\alpha}^{r}\right)_{|\alpha| \leq t_{r}, r=1, \ldots, k}\right)\right| \leq f(x)+L\left|\left(v_{\alpha}^{r}\right)_{|\alpha| \leq t_{r}, r=1, \ldots, k}\right|^{a} & \\
& \text { if }\left|v_{(0, \ldots, 0)}\right| \leq g(x) \text { a.e. } x .
\end{aligned}
$$

These assumptions give (13) and an a priori bound for solutions of the system (15) in the space $X_{r=1}^{k} \mathcal{H}^{t_{r}}$. The proof is similar to the one in Example 1 so it can be omitted.

\section{References}

[1] S. N. Bernstein, Sur les équations du calcul des variations, Ann. Sci. École Norm. Sup. 29 (1912), 431-485.

[2] F. E. Browder, Nonlinear functional analysis and nonlinear integral equations of Hammerstein and Urysohn type, in: Contributions to Nonlinear Functional Analysis, E. H. Zarantonello (ed.), Academic Press, New York 1971, 425-500.

[3] P. Fijalkowski, On the equation $x^{\prime \prime}(t)=F(t, x(t))$ in the Sobolev space $H^{1}(\mathbb{R})$, Ann. Polon. Math. 53 (1991), 29-34.

[4] A. Granas, R. Guenther and J. Lee, Nonlinear boundary value problems for ordinary differential equations, Dissertationes Math. 244 (1985).

[5] L. Hörmander, The Analysis of Linear Partial Differential Operators, Springer, Berlin 1983.

[6] M. A. Krasnosel'skiǔ, P. P. Zabreǔko, E. I. Pustyl'nik and P. E. Sobolevskiŭ, Integral Operators in Spaces of Summable Functions, Nauka, Moscow 1966 (in Russian).

[7] N. G. Lloyd, Degree Theory, Cambridge Univ. Press, 1978.

[8] B. Przeradzki, On the solvability of singular BVPs for second-order ordinary differential equations, Ann. Polon. Math. 50 (1990), 279-289.

INSTITUTE OF MATHEMATICS

UNIVERSITY OF ŁÓDŹ

BANACHA 22

90-238 ŁÓDŹ, POLAND 